\title{
Adaptação e validação de escalas de silhuetas para crianças brasileiras
}

\section{Adaptation and validation of silhouette scales for Brazilian children}

\author{
Elisa Pinheiro Ferrari ${ }^{1 *}$, Silvana Carolina Furstenau dos Santos ${ }^{1}$, Larissa Alves Maciel ${ }^{1}$, Diego \\ Soares Souza ${ }^{1}$, Julianna Mendes de Matos Souza ${ }^{1}$, Fernando Luiz Cardoso ${ }^{2}$
}

ARTIGO ORIGINAL | ORIGINAL ARTICLE

\begin{abstract}
RESUMO
O objetivo do presente estudo foi adaptar e testar a validade de constructo e a fidedignidade da "Children's Figure Rating Scale” para crianças brasileiras. Participaram do estudo 235 crianças, 120 meninos e 115 meninas, com média de idade de $8.8( \pm 1.1)$ anos. Além da aplicação da escala, foram realizadas medidas de massa corporal, e estatura para o cálculo do índice de massa corporal. Os dados foram analisados no programa Statistical Package for the Social Sciences ${ }^{\circledR}$ versão 20.0, a partir dos testes, correlação de Spearman, Wilcoxon e o Coeficiente de Correlação Intraclasse, uma vez que os dados não apresentaram distribuição normal de acordo com o teste de Kolmogorov Smirnov. Observou-se uma correlação positiva entre o índice de massa corporal e a imagem real e o primeiro com a discrepância para os meninos $(r=0.42 ; p=0.01)$ e meninas $(r=0.34 ; p=0.01)$. A fidedignidade foi confirmada por meio do coeficiente de correlação intraclasse, apresentando valores acima de $0.70(\mathrm{p}=0.001)$ para os dois sexos. Concluí-se que a escala testada demonstrou boas qualidades psicométricas, configurando-se como uma opção viável de avaliação da imagem corporal em crianças brasileiras.

Palavras-chave: imagem corporal, reprodutibilidade dos testes, criança.
\end{abstract}

\begin{abstract}
The aim of the present study was to adapt and test the construct validity and reliability of the "Children's Figure Rating Scale" for Brazilian children. The aim of the present study was to adapt and test the construct validity and reliability of the Children's Figure Rating Scale for Brazilian children. The sample consisted of 235 children, 120 boys and 115 girls, with a mean age of 8.8 ( \pm 1.1 ) years. Besides the scale, it was performed body mass and height to calculate the body mass index. The data were analysed in the Statistical Package for the Social Sciences, version 20.0, using the Spearman correlation, Wilcoxon Test and the Intraclass correlation coefficient, because the data did not present normal distribution according to the Kolmogorov Smirnov test. It was found a positive correlation between the body mass index and the real image for boys $(r=0.42 ; p=0.01)$ and girls $(r=0.34 ; p=0.01)$. The reliability was confirmed by the intraclass correlation coefficient, presenting values above $0.70(\mathrm{p}=0.001)$ for both, boys and girls. It was concluded that the scale tested has good psychometric qualities, being configured as a viable option of body image evaluation for Brazilian children.

Keywords: body image, results reproducibility, child.
\end{abstract}

\footnotetext{
${ }^{1}$ Universidade Católica de Brasília, Distrito Federal, Brasil.

${ }^{2}$ Universidade do Estado de Santa Catarina, Santa Catarina, Brasil.

* Autor correspondente: elisa.ferrari@ucb.br
} 


\section{INTRODUÇÃo}

A imagem corporal pode ser definida como uma ilustração mental acerca do tamanho, da aparência e da forma do corpo, assim como das respostas emocionais a ele associadas (Cash \& Pruzinsky, 1990). Atualmente há uma propagação de estereótipos de beleza e a impossibilidade de atender a estes modelos corporais pode induzir a uma imagem corporal negativa (Dunker \& Claudino, 2017).

$\mathrm{Na}$ população infantil tem se identificado um número crescente de crianças insatisfeitas com a sua imagem corporal as quais demonstram uma preocupação com a sua aparência física e uma necessidade de se enquadrar num padrão ideal de corpo (Steinsbekk et al., 2017). Evidências observadas a partir de um estudo de revisão apontam que a insatisfação com a imagem corporal aumenta com a idade (Ricciardelli \& McCabe, 2001) e que crianças com excesso de peso tendem a ser mais insatisfeitas que seus pares com peso normal ou magreza (Clark \& Tiggemann, 2006). Diante destes aspectos, a avaliação da imagem corporal se faz importante para identificar e diagnosticar quais grupos estão mais insatisfeitos com a sua imagem corporal.

Nesta temática, diversos instrumentos têm sido desenvolvidos para avaliar a imagem corporal infantil dentre eles, destaca-se a Children's Figure Rating Scale proposta por Tinggemann e Wilson Barret (1998), a qual consiste na apresentação de nove figuras, ordenadas da menor para maior em termos de tamanho corporal, sendo que os indivíduos são instruídos a escolher a que mais se assemelha a imagem que eles têm do seu próprio corpo e a que representa a sua imagem corporal ideal, caracterizando a insatisfação com a imagem corporal como a discrepância entre a imagem atual e a imagem ideal. Este tipo de instrumento vem sendo amplamente utilizado em pesquisas nacionais (Triches \& Giugliani, 2007; Pinheiro \& Jimenéz, 2010) devido a rapidez na aplicação, coleta e tabulação dos dados e a sua fácil utilização em diversos contextos culturais em comparação a outras medidas mais subjetivas (Kakeshita, Silva, Zanatta, \& Almeida, 2009).
A partir de uma revisão previsão prévia na literatura, foram identificadas a existência de instrumentos nacionais em forma de escala para avaliação da imagem corporal infantil (Kakeshita et al. 2009). Porém, segundo Borsa, Damásio e Bandeira (2012) a adaptação de um instrumento já existente, em detrimento da elaboração de um novo instrumento, possui vantagens consideráveis, maior capacidade de generalização e a investigação de diferenças entre uma crescente população diversificada. Diante do exposto, e da ampla popularidade do Children's Figure Rating Scale em estudos para avaliação da imagem corporal infantil e da inexistência de investigações que tratam de testar as propriedades psicométricas deste instrumento em crianças brasileiras, o objetivo do presente estudo foi adaptar e testar a validade de constructo e a fidedignidade da "Children's Figure Rating Scale” para criançcas brasileiras.

\section{MÉTODO}

Trata-se de um estudo transversal não probabilístico desenvolvido pelo Laboratório de Gênero, Educação, Sexualidade e Corporeidade (LAGESC) da Universidade do Estado de Santa Catarina (UDESC). Submetido e aprovado pelo Comitê de Ética em Pesquisas com Seres Humanos da UDESC sob o número de protocolo: 1.351.239/2015.

\section{Participantes}

A população do presente estudo foi limitada aos alunos com idades entre sete e 10 anos matriculados em três instituições públicas de ensino do município de Florianópolis, Santa Catarina. A seleção das instituições foi feita de forma intencional. Os participantes foram selecionados de maneira não probabilística, conforme a disponibilidade dos mesmos, para participação na pesquisa.

\section{Instrumentos e Procedimentos}

O Children's Figure Rating Scale é um instrumento desenvolvido por Tiggemann e Wilson-Barrett (1998) com o objetivo de avaliar a insatisfação com a imagem corporal em crianças e adolescentes. Esta escala é composta por nove 
figuras do sexo masculino e feminino, ordenadas da menor para a maior (Figura 1).
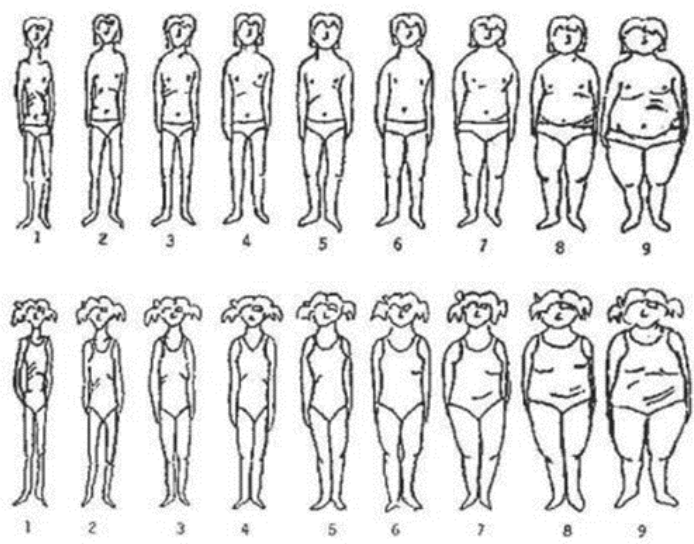

Figura 1. Escala Children's Figure Ratings

Para caracterizar o grau de insatisfação com a imagem corporal é subtraída a imagem indicada pelo sujeito como real pela ideal, sendo que a diferença igual a zero, indica satisfação com a imagem corporal e diferente de zero insatisfação, sendo valores positivos, insatisfação por excesso de peso e negativos, insatisfação por magreza.

A massa corporal e a estatura foram obtidas por meio de procedimentos padronizados (Ross \& Marfell-Jones, 1995). A partir destas, foi calculado o IMC, o qual utiliza a relação massa corporal $(\mathrm{kg})$ dividida pela estatura $(\mathrm{m})$ ao quadrado. Os valores de IMC foram classificados por meio dos pontos de corte estabelecidos por Cole et al. (2000; 2007), especificamente para crianças e adolescentes, considerando-se as categorias: magreza (magreza 1, 2 e 3), peso normal e excesso de peso (sobrepeso e obesidade).

Para verificar a reprodutibilidade da escala e a validade do construto da escala, a mesma foi aplicada em escolares e após um intervalo de sete a 10 dias foi reaplicada.

Após, a autorização do diretor da instituição de ensino, os estudantes foram contatados e apresentados aos objetivos do estudo mediante a entrega do Termo de Consentimento Livre e Esclarecido (TCLE).

A coleta de dados foi realizada na própria instituição de ensino, mediante a entrega do TCLE assinado pelos pais ou responsáveis. $\mathrm{Na}$ primeira coleta foram realizadas as medidas antropométricas e a aplicação do questionário. Após sete a 10 dias, o questionário era reaplicado nas mesmas condições da primeira coleta, por um pesquisador habilitado e treinado para a coleta de dados.

\section{Análise estatística}

Os dados foram analisados no programa Statistical Package for the Social Sciences (SPSS) ${ }^{\circledR}$ versão 20.0. Por meio dos valores de média, desvio-padrão, frequências relativas e absolutas. A distribuição dos dados foi verificada por meio do Teste de Kolmogorov-Smirnov, por não atender a condição de normalidade optou-se pela utilização dos testes não paramétricos. A fim de testar a validade de constructo foi realizada uma correlação de Spearman entre o valor do IMC e a imagem real. A fidedignidade foi avaliada por meio da comparação dos valores dos escores da escala de imagem corporal nos dois momentos da pesquisa (teste e reteste), utilizando o teste de Wilcoxon e o Coeficiente de Correlação Intraclasse. O nível de significância adotado para todas as análises foi de $5 \%$.

\section{RESULTADOS}

Foram avaliadas 236 crianças, com média de idade de 8.80 ( \pm 1.10 ) anos, sendo que $50.80 \%$ $(\mathrm{n}=120)$ pertenciam ao sexo masculino e $49.20 \%(n=116)$ pertenciam ao sexo feminino. Destas, 63 crianças participaram dos dois momentos, teste e reteste. Ao associar as categorias do IMC com as da imagem corporal, pode se verificar que para ambos os sexos, as crianças classificadas na categoria magreza e com excesso de peso foram as que apresentaram maior insatisfação por magreza e por excesso, respectivamente $(\mathrm{p}$-valor $=0.04)($ Tabela 1$)$. 
Tabela 1

Associação entre a classificação do IMC e da imagem corporal das crianças, Florianópolis, Brasil, 2017

\begin{tabular}{|c|c|c|c|c|}
\hline \multirow{3}{*}{ Categorias } & \multicolumn{4}{|c|}{ Sexo Masculino } \\
\hline & Satisf. & Insatisf. Excesso & Insatisf. magreza & \multirow{2}{*}{ p-valor } \\
\hline & $\%(\mathrm{n})$ & $\%(n)$ & $\%(\mathrm{n})$ & \\
\hline Magreza & $22.2(04)$ & $22.2(04)$ & $55.6(10)$ & - \\
\hline Peso normal & $46.1(35)$ & $25.0(19)$ & $28.9(22)$ & 0.001 \\
\hline Excesso de peso & $30.8(08)$ & $61.5(16)$ & $7.7(02)$ & - \\
\hline \multirow{3}{*}{ Categorias } & \multicolumn{4}{|c|}{ Sexo Feminino } \\
\hline & Satisf. & Insatisf. Excesso & Insatisf. magreza & \multirow{2}{*}{ p-valor } \\
\hline & $\%(\mathrm{n})$ & $\%(n)$ & $\%(\mathrm{n})$ & \\
\hline Magreza & $11.1(01)$ & $44.4(04)$ & $44.4(04)$ & - \\
\hline Peso normal & $45.5(35)$ & $40.3(31)$ & $14.3(11)$ & 0,002 \\
\hline Excesso de peso & $20.0(06)$ & $73.3(22)$ & $6.7(02)$ & - \\
\hline
\end{tabular}

Note. Satisf. = satisfeito; Insatisf. Excesso = insatisfeito por excesso; Insatisf. Magreza $=$ insatisfeito por magreza; $\%=$ frequência relativa; $\mathrm{n}=$ frequência absoluta.

Nas análises entre os escores da escala e os do IMC registraram-se correlações positivas e estatisticamente significantes para os meninos $(\mathrm{r}=0.42 \mathrm{p}=0.001)$ e meninas $(\mathrm{r}=0.34 \mathrm{p}=$ 0.001 ), sendo que quanto maior o IMC, maiores foram as figuras ideias reportadas (dados não apresentados em tabelas).

$\mathrm{Na}$ análise de fidedignidade ao se comparar os escores do teste e reteste, não foram observadas diferenças estatisticamente significantes tanto para os meninos, quanto para as meninas. Dentre os itens analisados, a figura ideal foi a que apresentou o menor percentual de concordância e CCI para o sexo masculino $(71.80 \%$; $\mathrm{CCI}=$ 0.93) e feminino (54.20\%; CCI $=0.77$ ) (Tabela 2).

Tabela 2

Associação entre a classificação do IMC e da imagem corporal das crianças, Florianópolis, Brasil, 2017

\begin{tabular}{|c|c|c|c|c|c|}
\hline \multirow{2}{*}{ Categorias } & Pré-teste & Reteste & Concordância & \multirow{2}{*}{$\mathrm{p}$} & \multirow{2}{*}{ CCI (IC95\%) } \\
\hline & $\mathrm{X}(\mathrm{DP})$ & $\mathrm{X}(\mathrm{DP})$ & $\mathrm{n}(\%)$ & & \\
\hline \multicolumn{6}{|c|}{ Sexo Masculino } \\
\hline Real & $4.64(1,70)$ & $4.56(1,66)$ & $18(75.0)$ & 0.4 & $0.97(0.94-0.98)$ \\
\hline Ideal & $4.58(1,33)$ & $4.48(1,44)$ & $28(71.8)$ & 0.4 & $0.93(0.87-0.96)$ \\
\hline \multicolumn{6}{|c|}{ Sexo Feminino } \\
\hline Real & $4.83(1.34)$ & $4.83(1.49)$ & $29(79.4)$ & 1.0 & $0.96(0.93-0.99)$ \\
\hline Ideal & $4.16(1.04)$ & $3.95(1.19)$ & $13(54.2)$ & 0.3 & $0.77(0.47-0.90)$ \\
\hline
\end{tabular}

Note. $\mathrm{X}=$ média; DP = desvio padrão; \% = frequência relativa; $\mathrm{n}$ = frequência absoluta; CCI (IC95\%) = Coeficiente de Correlação Intraclasse (intervalo de confiança de 95\%); Real = imagem real; Ideal = imagem ideal.

\section{DISCUSSÃO}

O processo de validação de um instrumento para outra cultura configura-se num procedimento necessário, principalmente quando se utiliza instrumentos referentes avaliação de aspectos psicológicos e a diversidade cultural dos seres humanos.

O presente estudo se propôs a testar a validade e a fidedignidade da Children's Figure Rating Scale (Tinggemann \& Wilson Barret, 1998) para o uso em crianças brasileiras. Esta técnica consiste num método de avaliação da imagem corporal infantil, amplamente utilizada em pesquisas nacionais. Desta forma, os dados levantados pela atual investigação são importantes, em virtude da escassez de estudos referentes as qualidades psicométricas dos instrumentos utilizados para avaliação da imagem corporal em crianças brasileiras.

Com relação à validade de constructo do instrumento em questão, testada a partir da hipótese da existência de uma correlação positiva entre o valor bruto do IMC o escore referente a imagem real. Os dados apresentados pelo 
presente estudo confirmam a validade de constructo da Children's Figure Rating Scale para crianças brasileiras de sete a 10 anos, mas os valores de correlação são inferiores aos apresentados pela literatura (Kakeshita et al., 2009; Adami et al., 2012). Ressalta-se que escala aplicada nestas investigações divergem da utilizada no presente estudo. Kakeshita et al. (2009) ao propor uma escala de silhuetas para a população infantil brasileira encontraram uma correlação positiva e significante entre o IMC da figura apontada como percepção atual e o IMC real das crianças tanto para meninas $(r=0.57)$ como meninos $(\mathrm{r}=0.64)$.

Adami et al. (2012) ao analisar a validade de constructo de uma escala de silhuetas para adolescentes encontraram valores de correlação entre a imagem corporal ideal e valores de score $\mathrm{Z}$ do IMC de 0.62 para os meninos e 0.54 para as meninas. Porém, ao correlacionar o escore $\mathrm{Z}$ do IMC com o escore da insatisfação só foram observadas correlações entre as variáveis para o sexo feminino, sendo que as meninas com scores $z$ mais altos de IMC também mostraram valores mais elevados de insatisfação com a imagem corporal.

A Children's Figure Rating Scale foi desenvolvida por Tinggemann e Wilson Barret (1998) de forma semelhante à proposta por Stunkard et at. (1983), porém voltada a população infantil. A escala de silhuetas de Stunkard foi validada para a população brasileira por Scagliusi et al. (2006) os quais encontraram uma correlação entre a figura real e o IMC de 0.76, o que indica que a escala utilizada para a população adulta brasileira e a escala adaptada para uso em crianças brasileiras são equivalentes, apesar de os valores de correlação não serem tão próximos.

Ao observar os resultados da análise de fidedignidade verifica-se que a mesma pode ser considerada boa, com valores de CCI que variaram de 0.77 a 0.97 , de acordo com o sexo. De forma geral, o item imagem real foi o que apresentou os maiores valores, acima de 0.95 para ambos os sexos. Estes resultados vão ao encontro dos observados em outros estudos que testaram a fidedignidade de escalas de silhuetas para avaliação da imagem corporal infantil, os quais observaram resultados do teste $t$ pareado, que não mostraram diferenças significativas entre os dados do teste e do reteste, reforçando a hipótese que crianças da faixa etária investigada tendem a ter capacidade de identificar o próprio tamanho corporal e desta forma, necessitam de instrumentos adequados e testados, a fim de suportar pesquisas no campo da imagem corporal infantil (Ricciardelli \& McCabe, 2001; Kakeshita et al., 2009).

Ao comparar os valores obtidos na análise de fidedignidade dos escores da silhueta ideal com a silhueta real, são encontrados valores inferiores de CCI para a silhueta ideal. Este desfecho corrobora ao estudo desenvolvido por Thompson, Heinberg, Altabe \& Tantleff-Dunn (1999) e pode ser explicado pela presença de fatores transitórios, dentre eles, a recente exposição a mensagens de mídia, o estado de humor, dentre outros, que podem afetar momentaneamente o julgamento do tamanho ideal (Thompson et al., 1999).

Como principal limitação destaca-se, a amostra por conveniência ser composta por estudantes de escolas de baixa vulnerabilidade social, o que impede a extrapolação dos dados para crianças de classes econômicas mais altas. No entanto, ressalta-se a importância dos resultados do presente estudo no fornecimento de informações acerca da validade psicométrica de uma escala de avaliação da imagem corporal infantil.

\section{CONCLUSÕES}

Diante dos resultados do presente estudo, pode-se concluir que a escala avaliada mostrou bom desempenho psicométrico quando examinada a partir de uma amostra de crianças brasileiras de um município do estado localizado no sul do Brasil.

Com a ampla utilização do instrumento em estudos nacionais, a disponibilidade deste, tende a favorecer o desenvolvimento de intervenções conferindo amplas possibilidades de utilização clínica e epidemiológica ao instrumento. Além disso, embora a escala tenha sido desenvolvida para a população australiana, os resultados encontrados com a sua aplicação confirmam a sua eficácia e fiabilidade para uso entre os brasileiros. 
Agradecimentos:

Nada a declarar

\section{Conflito de Interesses:}

Nada a declarar.

\section{Financiamento:}

Coordenação de Aperfeiçoamento de Pessoal de Nível Superior - CAPES.

Fundação de Apoio à Pesquisa Científica e Tecnológica do Estado de Santa Catarina - FAPESC.

\section{REFERÊNCIAS}

Adami, F., Frainer, D. E. S., Almeida, F. S., Abreu, L. C., Valenti, V. E., ... \& Oliveira, F. R. (2012). Construct Validity of a Figure Rating Scale for Brazilian Adolescents. Nutrition Journal, 11(24). doi: 10.1186/1475-2891-11-24

Borsa, J. C., Damásio, B. F. \& Bandeira, D. R. (2012). Adaptação e Validação de Instrumentos Psicológicos Entre Culturas: Algumas Considerações. Paideia, 22 (53), 423-432. doi: 10.1590/1982-43272253201314

Cash, T. F., \& Pruzinsky, T. (1990). Body Images. Development, Deviance and Change. NY and London: The Guilford Press.

Clark, L., \& Tiggemann, M. (2006). Appearance Culture in Nine- to 12-Year-Old Girls: Media and Peer Influences on Body Dissatisfaction. Social Development, 15(4), 628-643. doi: 10.1111/j.14679507.2006.00361.x.

Cole, T. J. (2000). Establishing a Standard Definition for Child Overweight and Obesity Worldwide: International Survey. Bmi, 320(7244), 1240-1243. doi:10.1136/bmj.320.7244.1240

Cole, T. J., Flegal, K. M., Nicholls, D., \& Jackson, A. A. (2007). Body Mass Index Cut Offs to Define Thinness in Children and Adolescents: International Survey. British Medical Journal, 335(7612), 194-197. doi: 10.1136/bmj.39238.399444.55

Dunker, K. L. L., \& Claudino, A. M. (2017). Validity and Reliability of the Brazilian Version of the Weight Control Behaviors Scale. Journal of Pediatrics, 189, 143-148. doi: 10.1016/j.jpeds.2017.06.037
Kakeshita, I. S., Silva A. I., Zanata, D. P., \& Almeida, S. S. (2009). Construção e Fidedignidade TesteReteste de Escalas de Silhuetas Brasileiras para Adultos e Crianças. Psicologia: Teoria e Pesquisa, 25(2),63-70. doi: 10.1590/S010237722009000200015

Pinheiro, N., \& Jiménez, M. (2010). Percepção e Insatisfação Corporal: Um Estudo Em Crianças Brasileiras. Psico, 41 (4), 510-516.

Ricciardelli, L. A., \& McCabe, M. P. (2001). Children's Body Image Concerns and Eating Disturbance: A Review of the Literature. Clinical Psychology Review, 21(3), 325-344. doi: 10.1016/S02727358(99)00051-3

Ross, W. D., \& Marfell-Jones, M. J. (1995). Cineantropometria. In H. J. Green, H. A. Wenger, \& D. J. Mac Dougall (Eds.), Evaluación Fisiologica Del Deportista (3 a ed., pp. 233-306). Barcelona: Editorial Pai da Tribo.

Scagliusi, F. B., Alvarenga, M., Polacow, V. O., Cordás, T. A., Queiroz, G. K. O, \& Coelho, D. (2006). Concurrent and Discriminate Validity of the Stunkard's Figure Rating Scale Adapted into Portuguese. Appetite, 47(1), 77-82. doi: 10.1016/j.appet.2006.02.010

Steinsbekk, S., Klockner, C. A., Fildes, A., Rognsas, P. L. S., \& Wichstrom, L. (2017). Body Size Estimation from Early to Middle Childhood: Stability of Underestimation, BMI, and Gender Effects. Frontiers in Psychology, 21(8). doi: 10.3389/fpsyg.2017.02038. eCollection 2017

Stunkard, A., Sorensen, T. F. S., \& Schulsinger. F. (1983). Use of the Danish Adoption Register for the Study of Obesity and Thinness. In: Kety, S. S., Sidman, L. P., \& Matthysse, R. L. The Genetics of Neurological and Psychiatric Disorders (pp. 115120). New York: Raven Press.

Thompson, J. K., Heinberg, L. J., Altabe, M. N., \& Tantleff-Dunn, S. (1999). Exacting beauty: Theory, assessment and treatment of body image disturbance. Washington, DC: American Psychological Association.

Tiggemann, M., \& Wilson-Barret, E. (1998). Children's Figure Ratings: Relationship to Self-Esteem and Negative Stereotyping. International Journal of Eating Disorders, 23(1), 83-88. doi: 10.1002/(SICI) 1098-108X

Triches, R. M., \& Giugliani, E. R. J. (2007). Insatisfação Corporal em Escolares de dois Municípios Da Região Sul Do Brasil. Revista de Nutrição, 20(2), 119-128. doi: 10.1590/S141552732007000200001 